7. Active Galactic Nuclei, Cosmic X-ray Background 


\title{
HIGH RESOLUTION X-RAY SPECTROSCOPY OF ACTIVE GALACTIC NUCLEI
}

\author{
Julian H. Krolik \\ Johns Hopkins University, Baltimore MD, USA
}

\begin{abstract}
High-resolution X-ray spectroscopy has the potential to reveal a number of interesting features of active galactic nuclei, primarily, though not exclusively, through the measurement of absorption lines. After a brief review of the principal problems of AGN research, selected potential high-resolution observations are discussed with a view toward assessing their scientific value and the degree of resolution they will require. Two classes of observations pertaining directly to AGNs are discussed: Fe $\mathrm{K} \alpha$ spectroscopy relevant to the dynamical and thermal character of the emission line zones; and measurement of resonance line absorption by highly-ionized species in BL Lac objects, which should tell us about entrainment of interstellar material by relativistic jets. A third class of potentially important observations uses AGNs as background light sources in order to directly measure the distance to clusters of galaxies.
\end{abstract}

\section{OVERVIEW OF ACTIVE GALACTIC NUCLEI}

\section{1. Salient Observational Features}

The single most striking attribute of AGNs is the extraordinary luminosity they are capable of generating. The current record is $10^{48} \mathrm{erg} \mathrm{s}^{-1}$ (Kühr, et al. 1983), i.e., $\sim 10^{5} \times$ the luminosity of a typical galaxy. Although in many objects there is a local peak in the luminosity per logarithmic bandwidth near $2.5 \times 10^{15} \mathrm{~Hz}$, this peak generally accounts for only $\simeq 20 \%$ of the total luminosity, the rest being spread almost evenly in $\log \nu$ from $\sim 10^{11}$ to $\sim 10^{20} \mathrm{~Hz}$ (e.g., Ward, et al. 1987). Moreover, substantial variations in this large power output are seen, sometimes over periods as short as hours or days, and often over longer timescales. The combination of extremely broad-band emission and very large power produced in a very small volume makes us quite confident that something other than an assembly of stars is responsible for this phenomenon.

In addition to these attention-grabbers, two other properties attract our interest: their strong cosmological evolution, and the presence of very high velocity 
gas at relatively low temperature. In the last year or so, enough survey data has been accumulated that we now have a reasonably clear picture of the evolution of the brighter AGNs back to $z \simeq 2.5$ (Boyle, et al. 1987; Koo and Kron 1988): the most luminous AGNs were many orders of magnitude more common (in co-moving density) then than now. Somehow the adolescence of galaxies was especially conducive to nuclear activity.

The most prominent features of the optical and UV spectra of AGNs are the strong emission lines: these frequently double the specific flux, and extend over as much as $10,000 \mathrm{~km} \mathrm{~s}^{-1}$. Studies of their relative strengths have shown that the temperature of the emitting gas is in the range $1-2 \times 10^{4} \mathrm{~K}$, so that the Mach number of the gas's motion is $\sim 10^{3}$ ! How such quantities of cool gas are accelerated to such high speeds is still quite mysterious (e.g. as reviewed by Mathews and Capriotti 1985).

\section{2. Consensus Theoretical Cartoon}

Some twenty-five years of work has led to a consensus view of AGN structure which I caricature in Fig. 1. At the center lies a massive black hole $(M \sim$ $10^{8}\left(L_{E} / L\right) L_{46} M_{\odot}$, where the luminosity is referred both to the Eddington luminosity and to the convenient unit $10^{46} \mathrm{erg} \mathrm{s}^{-1}$ ) into which material drops at the rate $\dot{M} \sim 1 L_{46} \mathrm{M}_{\odot} \mathrm{yr}^{-1}$ if the efficiency of transforming fuel into radiated power is $\sim 0.1$, as might be expected from material accreting with non-zero angular momentum. The extent of the accretion disk is very hard to determine, which is why its shading in the figure is shown trailing off with increasing radius. Photoionization modelling (Davidson and Netzer 1979; Kwan and Krolik 1981; Ferland and Mushotzky 1982) argues that the emission lines with the largest velocities are made $\sim 1 L_{46}^{1 / 2} \mathrm{pc}$ from the source of the ionizing continuum (this is the "broad line region") while the lines of smaller width $\left(\sim 300-500 \mathrm{~km} \mathrm{~s}^{-1}\right)$ are made in a region $\sim 100-1000 \times$ farther away (the "narrow line region"); variability monitoring of one object, NGC 4151, has recently (weakly) confirmed this distance estimate for the high velocity gas (Clavel, et al. 1987; Edelson and Krolik 1988).

The only change to this picture made in recent years is the discovery of the "intermediate zone". In a tour de force of spectropolarimetry, Antonucci and Miller (1985) demonstrated that in the archetype (NGC 1068) of a particular sub-class of AGN (type 2 Seyfert galaxies) an extremely optically thick torus blocks our line of sight to the central continuum source and the high-velocity emission line gas, so that the only way we can detect the true nucleus is through the polarization imparted to the light reflected off warm plasma which fills the hole of the torus and extends some ways above its top. More recent work reported by Miller (108S) suggests that this structure is indeed generic.

Given these properties and supposed structure, several questions immediately demand answers. First, is the energy source truly accretion, and if so, how does the 


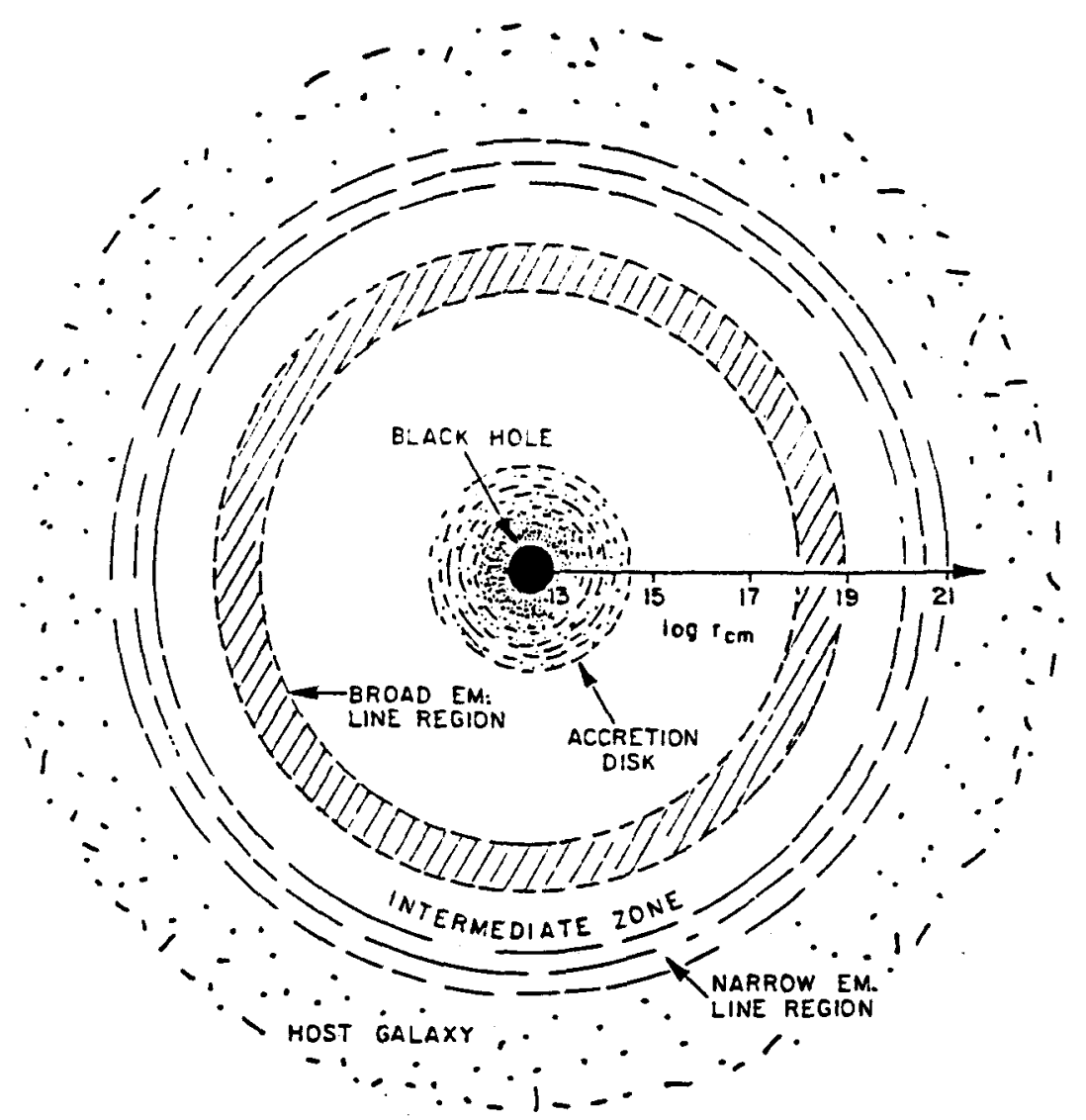

Figure 1. A sketch of the shell structure of AGN as it is envisaged by most workers in the field.

material travel to the black hole? Although $1 \mathrm{M}_{\odot} \mathrm{yr}^{-1}$ is not a huge amount of mass loss for all the stars in a galaxy to toss off, $100 \mathrm{M}_{\odot} \mathrm{yr}^{-1}$ is much harder to envisage, and in practise one might expect much greater requirements when all the likely inefficiencies are taken into account. The most important of the likely inefficiencies is, of course, the difficulty of removing all but $10^{-5}$ of the gas's angular momentum. We would very much like to understand how that happens. A prerequisite of that inquiry is, of course, to infer the physical conditions in which the accretion flow takes place.

Second, how are the photons generated? The simplest plausible picture of an accretion flow is that of an optically thick, geometrically thin disk (Lynden-Bell and Pringle 1974; Shakura and Sunyaev 1973) in which the state of thermodynamic equilibrium obviates any necessity to isolate the specific mechanisms of photon production. In fact, the notorious "blue bump" found in so many AGNs lends support to this picture, for its characteristic energy $(\sim 10 \mathrm{eV})$ is right in line with what might be expected a priori (Sun and Malkan 1988; Wandel and Petrosian 19S8). 
Unfortunately, fits to the spectra of some objects suggest physical inconsistencies (most notably, sometimes $L / L_{E}$ becomes large enough to make the thin disk approximation inapplicable), and the majority of the light, as remarked above, is not contained in the blue bump, and appears to be radiated by some genuinely nonthermal mechanism. Our usual repertory of non-thermal mechanisms (synchrotron radiation, inverse Compton scattering) generally requires relativistic electrons; what is their source?

Third, just what is the connection to young galaxies? The growing evidence for a correlation (albeit imperfect) between unusually high rates of star formation and nuclear activity in galaxies of the present epoch suggests that young may mean both chronologically and evolutionarily. For example, it may be that having large quantities of gas is the important element of "youth", or it may also be that recent dynamical disturbance is the important thing.

Fourth, what is the origin of variability? When the variability seems especially violent and rapid (those AGNs exhibiting this sort of behavior are often labelled "blazars"), relativistic jets directed toward us are often invoked. Is there any way to study these jets directly?

Finally, though it is certainly not the last question one could ask, what are the forces which accelerate the cool gas to such high speeds? Radiation pressure, gravity, and drag against an external fluid medium have all been suggested, but none has clearly won the day.

By citing just a few examples in the next several pages, I hope to demonstrate that high-resolution X-ray spectroscopy has something to contribute towards answering all these questions.

\section{NARROW FEATURES INTRINSIC TO X-RAY PRODUCTION}

The strong suggestion that most of the continuum (outside the UV) is generated non-thermally also implies that-at least in the simplest picture-there is probably little fine-scale structure intrinsic to the $\mathrm{X}$-ray continuum. If relativistic electrons (and maybe positrons) really are responsible for generating the $\mathrm{X}$-ray photons, the only feature one might expect would be a very broad one associated with $m_{e} c^{2}=511 \mathrm{keV}$. Even if narrow features are somehow present in the spectrum emitted from individual small regions, the large velocities associated with the neighborhood of a black hole are likely to spread out these featuresd over a range $\delta \nu / \nu \sim 0.1$ in the composite spectrum. Thus, if any narrow X-ray features exist, the simplest theoretical prediction is that they are likely to be created in regions of smaller velocity farther away from the center of activity, and their interpretation will provide relatively little guidance in understanding $\mathrm{X}$-ray production.

However, the arguments of the preceding paragraph are far from water-tight. Some have argued that thermal material should always be present, even in regions of predominantly non-thermal radiation (Guilbert and Rees 1988). If that is the 
case, absorption by relatively cool material may provide diagnostics of the pressure and velocity in the region of $\mathrm{X}$-ray radiation. In Saha equilibrium, for example, temperatures as high as $\sim 100 \mathrm{eV} \sim 10^{6} \mathrm{~K}$ still permit some $\mathrm{H}$-like $\mathrm{Si}$ and $\mathrm{S}$, while Fe still retains half a dozen electrons. The thermal widths of lines in these conditions are extremely small-fractions of $1 \mathrm{eV}$ - so that the line width is a direct indicator of dynamics in the absorbing region, while the specific ionic stage responsible gives information about the thermal state of the material.

\section{Fe K $\alpha$ FROM EMISSION LINE REGIONS}

\section{1. The Broad Line Region}

There are several places within the various emission line regions likely to produce narrow $\mathrm{Fe} \mathrm{K} \alpha$ features. I will discuss them starting on the inside (the broad line region) and moving out. A fair amount is known about the material which radiates the broad lines: most of its $\mathrm{Fe}$ is singly-ionized, and the total column is at least $\sim 10^{23} \mathrm{H} \mathrm{cm}^{-2}$ on average. We can therefore expect hard X-rays striking it to produce fluorescent $\mathrm{K} \alpha$ photons spread from 6.38 to $6.42 \mathrm{keV}$ by the same motions that broaden the optical and UV lines. The equivalent width of this feature is proportional to the covering factor of this material over the $\mathrm{X}$-ray source; photoionization models which predict optical/UV line emissivities yield values of $C$ for different objects ranging from 0.01 up to nearly unity, and so we might expect in a typical source to see equivalent widths anywhere from 6 to $600 \mathrm{eV}$. In fact, turning this around, measuring the equivalent width of this cold Fe fluorescent $\mathrm{K} \alpha$ line would be very useful, for it would provide an estimate of $C$ which would be much more nearly model-independent. A resolved line observation would be that much more specific, giving $d C / d v_{r}$ where $v_{r}$ is the velocity projected on the line of sight.

Even when $C \sim 1$, the volume filling factor of the line-emitting gas is small. This fact has led many people to imagine that the line-emitting gas is contained within a large number of small clouds, surrounded and confined by a much hotter, much less dense medium. This putative intercloud medium is thought to have in temperature of at least $10^{8} \mathrm{~K}$ and a column density of $\sim 10^{24} L_{46}^{1 / 2} T_{8}^{-1 / 2} \mathrm{H} \mathrm{cm}^{-2}$ (Krolik, McKee, and Tarter 1981), but we have no direct indication of its existence, or, if it cloes exist, of its state of motion. Kallman and Mushotzky (19S5) pointed out that if $T$ is not too much greater than $10^{8} \mathrm{~K}$, the intercloud medium should produce a $\mathrm{P}$ Cygni (or reverse P Cygni) profile in scattered Fe XXVI K $\alpha$. For example, if the intercloud medium moves at a speed comparable to the clouds' speeds, there would be a feature of optical depth order unity stretching from $\simeq 6.7$ to $\simeq 7.1 \mathrm{keV}$.

Detection of such a feature would teach us a great deal. Even an upper limit would give a constraint on its pressure formed by an upper bound on its density and a lower bound on its temperature. Detection would, of course, confirm the 
existence of the intercloud medium, and provide a fairly good description of its ionization state and density. But beyond that, we would also learn both the sign and magnitude of its velocity. If its speed matches that of the line-emitting material, then we will instantly understand how the cool gas came to move so fast. If it turns out to be flowing inward, there would be strong grounds for identifying it with the accretion flow, and for concluding that accretion is quite inefficient, for the likely magnitude of the mass flow it carries is $\sim 1000 L_{46} T_{8}^{-1}\left(v / 10^{4} \mathrm{~km} \mathrm{~s}^{-1}\right) M_{\odot} \mathrm{yr}^{-1}$.

\section{2. The Intermediate Zone}

The second emission line zone from which an interesting Fe $\mathrm{K}$ feature can be expected is the intermediate zone, where the host galaxy and the active nucleus have their dynamical interface. It is reasonable to suppose that the accretion mechanics are controlled by whichever system dominates the local gravity; the bounclary between the central black hole and the host galaxy occurs between the broad and the narrow emission line regions over a wide range of $L / L_{E}$ and $\mathrm{L}$. This clomain is also precisely the zone in which the obscuring torus and reflecting plasma described in $\S 1$ can be found. In fact, theoretical analysis (Krolik and Begelman 1988) has shown that the dynamics of both the torus and the reflecting plasma probably play an important role in regulating the accretion into the nucleus. Because the torus is probably composed of a large number of dusty molecular clouds which sift inward on a timescale not greatly longer than the orbital period, its very existence depends on having an adequate reservoir of molecular gas farther out in the host galaxy, and on having a sufficient level of dynamical disturbance in that reservoir to bring in the gas necessary to resupply the torus. The considerable geometrical thickness of the torus (Seyfert galaxy statistics require its height to be almost as large as its cylindrical radius) then implies that these clouds have a significant vertical velocity dispersion. Whatever mechanism stirs the torus material hard enough to explain its geometrical thickness is also likely to create a distribution of specific angular momentum broad enough to allow the material with the least angular momentum to be captured by the nucleus. Moreover, unless a fraction of the mass inflow through the torus does contribute to the accretion luminosity, the position of the torus's inner edge is unstable. Krolik and Begelman (1986) showed that the reflecting plasma is created by photoionization evaporation of the inner edge of the torus, so the capture fraction depends on its dynamics.

Fe $\mathrm{K}$ spectroscopy may help us understand this regulation of accretion because the reflecting plasma should produce sizable features both in fluorescent $K a$ and in the K-edge (Krolik and Kallman 1987). From estimates of the fraction of the nuclear light reflected and the total luminosity of the nucleus, it is possible to predict the ionization state of the plasma: Fe XVII - Fe XXIII. The ratio of K-edge to Thomson opacity is nearly independent of physical conditions, so the equivalent width of the fluorescent $\mathrm{K} \alpha$ from this gas, when viewed in the equatorial plane of the 
torus, is $\simeq 0.5[\mathrm{Fe} / \mathrm{H}] /[\mathrm{Fe} / \mathrm{H}]_{\odot} \mathrm{keV}$, essentially independent of all variables but the $\mathrm{Fe}$ abundance. In fact, just such a line has now been detected by Ginga (Iioyama 1988). With sufficient resolution, this line should break up into half a dozen components with central energies ranging from 6.50 to $6.70 \mathrm{keV}$, each betraying the abundance of a particular ionization stage, and each offset and broadened by a few $\mathrm{eV}$ due to bulk and thermal speeds $\sim 100 \mathrm{~km} \mathrm{~s}^{-1}$. The same galaxies viewed along the toroidal axis would produce a $\mathrm{K} \alpha$ line of roughly ten times smaller equivalent width, and a K-edge of optical depth $\sim 0.1$.

Spectra which resolved these features would allow a very precise determination of the temperature and density of the reflecting plasma, as well as its velocity. From these quantities, much about the capture if accretion fuel by the central engine could be learned.

\section{ABSORPTION LINES IN BLAZARS}

We already know of one example of a narrow absorption feature appearing in a blazar: using the OGS on the Einstein Observatory, Canizares and Kruper (1084) found an absorption line of optical depth $\simeq 3$ stretching from $\simeq 650$ to $\simeq 750 \mathrm{eV}$ (in the rest-frame) in the BL Lac object PKS 2155-304. In four other BL Lac objects, there are hints of similar absorption in the coarser-resolution SSS data, but they were too faint to observe with the OGS (Urry, Mushotzky, and Holt 1986). No information exists on "optically violently variable" quasars, which, along with BL Lac objects, comprise the class of blazars. Clearly, greater sensitivity in high resolution spectroscopy is a priority for this class of objects.

The interpretation of this absorption is quite controversial. The observed energy matches OVIII L $\alpha$ quite nicely if we are only seeing material moving towards us. The mass loss rate and energy associated with such an outflow are dauntingly large if it is spherically symmetric: $\dot{M} \simeq 2700 M_{\odot} \mathrm{yr}^{-1}$ and $L_{k i n} \simeq 10^{48} \mathrm{erg} \mathrm{s}^{-1}$ (Krolili, et al. 1985). On the other hand, beaming might well be expected in BL Lac objects, and we would only see them when the beam is directed towards us. The problem then is in explaining why this material has a maximum velocity $\sim 0.1 c$ when the velocity of the beamed material producing the photon luminosity should be very near $c$ in order to successfully explain the extreme variability of these objects. Perhaps the absorption is due to ordinary interstellar matter entrained along the outer edge of the jet? If so, can its study teach us about jet mechanics?

More high resolution measurements of features like these would be very helpful. We need to learn, for a start, whether this really is a general phenomenon. Detection of absorption in other species would also be an important clue, for the mass loss estimates derived by Krolik, et al. (1985) depend on the ionization balance of the gas. It would be particularly interesting, for example, if absorption by other jons were detected, to see whether they are present over the same range of velocitics 
as OVIII. Unfortunately, identification of lines could be complicated if there is evidence for substantial differences in their velocities; Doppler shifts are automatically substantial when the motions are even as close to relativistic as these.

\section{ABSORPTION LINES IN GALAXY CLUSTERS}

As Sarazin discusses at greater length in this volume, clusters of galaxies in general contain substantial quantities (column density $N \sim 10^{22} \mathrm{H} \mathrm{cm}^{-2}$ ) of hot $\left(T \sim 10^{7}-10^{8} \mathrm{~K}\right)$ gas. Most elements are totally stripped of electrons in such an environment, but several high- $\mathrm{Z}$ species, such as $\mathrm{Si}, \mathrm{S}, \mathrm{Ca}, \mathrm{Ar}$, and $\mathrm{Fe}$, still retain a few. Given the typical column density, resonance transitions in these ions should produce absorption lines of equivalent width $\sim 1-20 \mathrm{eV}$ in the spectrum of any X-ray continuum source, e.g. a background quasar, which happens to be lined up behind the cluster. Depending on the temperature and actual column density through a given cluster, anywhere from two to a dozen lines should have significant equivalent widths. These have considerable intrinsic interest for the study of elemental abundances, temperature structure within the gas, and dynamics of both the gas and galaxies in the cluster, but I wish to focus attention here on how they may be used for another purpose: to measure the angular diameter distance from us to the cluster, and thereby calibrate the Hubble ratio and measure qo (Krolik and Raymond 1988).

The basic idea is very similar to a decade-old proposal (Gunn 1978; Silk and White 1978; Cavaliere, et al. 1979) to measure the distances of galaxy clusters, but removes the principal stumbling block which has hindered its implementation. Stripped to its bare essentials, the scheme is to obtain a column density $n l$ by measurement of an absorption feature, while measurement of an optically thin emission feature gives an emission measure $n^{2} l$. If the line of sight lengthscale $l$ is the same as the transverse lengthscale, the angular diameter distance is:

$$
D_{A} \simeq \frac{(n l)^{2}}{\left(n^{2} l\right) \theta}
$$

where $\theta$ is the angular size of the hot gas in the cluster.

In the old version, the absorption measurement was the reduction in intensity of the microwave background in the direction of the cluster due to the SunyaevZel'dovich effect. Its expected magnitude is $\Delta I / I \sim n l \sigma_{T}\left(k T / m_{e} c^{2}\right) \sim 10^{-4}$. The temperature weighting in $\Delta I / I$ can be removed by fitting temperature-clependent emissivity models to the total flux spectrum of the hot gas. The emission measure was to be derived from the $\mathrm{X}$-ray continuum surface brightness. The central problem which has prevented the implementation of this scheme is the difficulty of measuring $\Delta I / I$ (see, e.g., Birkinshaw, Gull, and Hardebeck 1984).

In the new version, the absorption feature is any of the X-ray absorption lines described at the beginning of this section, while the emission measure can be 
taken from either the continuum surface brightness, or the surface brightness of these same lines as they appear in emission away from the background quasar. To get from the column density in a particular ion to the hydrogen column density, one uses the abundance in that ion defined by the strength of its forbidden or subsidiary emission lines. For each resonance line, one then has two independent distance determinations.

This new method has many advantages over the old version. Provided one has an instrument capable of resolving the lines, they are easy to detect, in contrast to the Sunyaev-Zel'dovich effect whose intrinsic smallness makes it difficult to reliably detect independent of instrument. Furthermore, the large number of lines which should be measurable makes it possible to severely control the error of the distance determination: in a typical case, one is likely to have $\sim O(10)$ independent measurements of the distance to a given cluster!

The only drawback to this scheme is that it depends on having an aligned quasar bright enough to measure accurately behind any cluster whose distance is desired. While it is true that even with the sensitivity of AXAF, such a quasar can be found in only a small minority of clusters, there should still be enough clusters with aligned bright quasars-probably something like several dozen-- to permit independent measurements of both $H_{o}$ and $q_{o}$.

\section{CONCLUSIONS}

Although I have presented only a small sample of the sorts of observations which could be done with high-resolution X-ray instrumentation, I hope I have demonstrated their substantial interest. Finding narrow features intrinsic to the $\mathrm{X}$-ray continuum is something of a long-shot, but if they are present, they could be extremely helpful in understanding the physical conditions prevalent in the region of continuum radiation. Fe $\mathrm{K}$ spectroscopy, however, is virtually a sure bet, and has ramifications ranging from the dynamics of the broad emission line region to the regulation of the accretion flow and the evolution of nuclear activity. Beyond these matters of intrinsic interest to AGNs, high resolution X-ray spectroscopy applied to active galactic nuclei can also be used to make measurements of the fundamental geometric quantities of the Universe.

ACKNOWLEDGMENTS. I am grateful to Craig Sarazin for a conversation at this meeting which helped clarify the role emission line measurements play in defining the heavy element abundances in intra-cluster gas. This work was partially supported by NASA Grant NAGW-1017. 


\section{REFERENCES}

Antonucci, R.R.J. and Miller, J.S., 1985, Ap. J. 297, 621.

Birkinshaw, M., Gull, S.F., and Hardebeck, H., 1984, Nature 309, 34.

Boyle, B.J., Fong, R., Shanks, T., and Peterson, B.A., 1987, M.N.R.A.S. 227, 717.

Canizares, C. and Kruper, J., 1984, Ap. J. Lett. 278, L99.

Cavaliere, A., Danese, L., and De Zotti, G., 1979, Astr. Ap. 75, 322.

Clavel, J., al., 1987, Ap. J. 321, 251.

Davidson, K. and Netzer, H., 1979, Rev. Mod. Phys. 61, 715.

Edelson, R.A. and Krolik, J.H., 1988, Ap. J. 333, 646.

Ferland, G. and Mushotzky, R.F., 1982, Ap. J. 262, 564.

Guilbert, P.W. and Rees, M.J., 1988, M.N.R.A.S. 233, 475.

Gunn, J.E., 1978, in Observational Cosmology, A. Maeder, L. Martinet, and G. Tammann, eds. (Geneva: Geneva Observatory).

Kallman, T.R. and Mushotzky, R.F., 1985, Ap. J. 292, 49.

Koo, D. and Kron, R., 1988, Ap. J. 325, 92.

Koyama, K., 1989, in IAU Symposium 194: Active Galactic Nuclei, D. Osterbrock and J.S. Miller, eds. (Reidel: Dordrecht)

Krolik, J.H. and Begelman, M.C., 1986, Ap. J. Lett. , 308, L55.

Krolik, J.H. and Begelman, M.C., 1988, Ap. J. in press.

Krolik, J.H. and Kallman, T.R., 1987, Ap. J. Lett. 320, L5.

Krolik, J.H., Kallman, T.R., Fabian, A.C., and Rees, M.J., 1985, Ap. J. 295, 104.

Krolik, J.H., McKee, C.F., and Tarter, C.B., 1981, Ap. J. 249, 422.

Krolik, J.H. and Raymond, J.C., 1988, Ap. J. Lett. in press.

Kühr, H., Liebert, J.W., Strittmater, P.A., Schmidt, O.D., Mackay, C., 1983, Ap. J. Lett. 275, L33.

Kwan, J.Y. and Krolik, J.H., 1981, Ap. J. 250, 478.

Lynden-Bell, D. and Pringle, J., 1974, M.N.R.A.S. 168, 603.

Mathews, W.G. and Capriotti, E.R., 1985, in Astrophysics of Active Galaxies and Quasi-Stellar Objects, ed. J.S. Miller (University Science Books: Mill Valley CA)

Miller, J.S., 1988, in Active Galactic Nuclei, H.R. Miller and P.J. Wiita, eds. (Rcidel: Dordrecht)

Shakura, N.I. and Sunyaev, R.A., 1973, Astr. Ap. 24, 337.

Silk, J. and White, S.D.M., 1978, Ap. J. Lett. 226, L103.

Sun, W.-H. and Malkan, M.A., 1988, preprint

Urry, C.M., Mushotzky, R.F., and Holt, S.S., 1986, Ap. J. 305, 369.

Wandel, A. and Petrosian, V., 1988, Ap. J. Lett. 329, L11.

Ward, M., Elvis, M., Fabbiano, G., Carleton, N., Willner, S., and Lawrence, A., 1987, Ap. J. 315, 74.

Zdziarski, A.A., 1986, Ap. J. 305, 45. 
DISCUSSION-J. Krolik

S. Kahn: Isn't OVIII L $\alpha$ also a good candidate for absorption line studies through clusters? I would have expected that it would have appreciable optical depth.

J. Krolik: At temperatures above $10^{7} \mathrm{~K} \mathrm{O}$ is pretty thoroughly stripped. Probably you are thinking of the very center of the hypothetical cooling flow, where the temperature is low enough to permit some $\mathrm{O}$ to recombine. Although the emission measure in this region can be substantial, it doesn't occupy enough solid angle to be a likely target for absorption line studies-except for cases like M87 where an $\mathrm{x}$-ray continuum source sits right in the middle of a cooling flow.

C. Sarazin: I would think that Broad Absorption Line Quasars would be ideal candidates for X-ray absorption lines. Any comments?

J. Krolik: You're absolutely right. Although their K-edge opacity should produce a feature of only small contrast, the species observed in them are highly-enough ionized (Li-like) that $\mathrm{K} \alpha$ lines should be present and quite optically thick. Unfortunately, I don't see that the information we gain by observing them will add qualitatively from what we already have from the UV resonance lines. 